\title{
Graphical and Database Analysis of New Sequence of Functions Involving the Bessel Function with MatLab Implementation
}

\author{
Jaspreet Kaur \\ Department of Computer Science \\ Baba Farid Group of Institutions \\ Bathinda-151001, India
}

\author{
Ranbir Kaur Brar \\ Department of Computer Science \\ Baba Farid Group of Institutions \\ Bathinda-151001, India \\ Mehar Chand \\ Department of Mathematics \\ Malwa college of IT and \\ Managment \\ Bathinda-151001, India
}

\author{
Kanwarjit Singh \\ Department of Computer Science \\ T.P.D. Malwa College \\ Rampura Phul
}

Bathinda-151103, India

\begin{abstract}
The aim of the present paper is an attempt to introduce a new sequence of functions $\left\{V_{n}^{(v, \alpha)}(x ; a, k, s) / n=0,1,2, \ldots\right\}$, which involving the Bessel function of first kind $J_{v}(x)$. By using operational technique, some interesting generating relations and summation formulas are obtained in sections 2 and 3 . The remarkable thing of this paper is the crucial MATLAB coding of the new sequence of functions, Database and Graph established using the MATLAB (R2012a) in the section (5) and (6) for different values of parameters and $n=1,2,3$ and 4. The reader can establish Database and Graph using the same program for any value of $n$.
\end{abstract}

\section{General Terms}

Special function, Sequence of Function, Matlab.

\section{Keywords}

Special Function, Generating function, Bessel function of first kind, Sequence of function, Matlab.

\section{INTRODUCTION}

In the present Era, MATLAB is a high-performance language for technical computing. It integrates computation, visualization, and programming in an easy-to-use environment where problems and solutions are expressed in familiar mathematical notation. Typical uses include:

- Math and computation

- Algorithm development

- Modeling, simulation, and prototyping

- Data analysis, exploration, and visualization

- Scientific and engineering graphics

- Application development, including Graphical User Interface building

In pure mathematics, since Matlab is an integrated computer software which has three functions: symbolic computing, numerical computing and graphics drawing. Matlab is capable to carry out many functions including computing polynomials and rational polynomials, solving equations and computing many kind of mathematical expressions. One can also use Matlab to calculate the limit, derivative, integral and Taylor series of some mathematical expressions. With Matlab, The graphs of functions with one or two variables can be easily drawn in selected domain. Therefore, functions can be studied by visualization for their main Characteristics. Matlab is also a system which can be easily expanded. Matlab provides many powerful software packages which can be easily incorporated into the clients system. Recently, there are many papers in the literature which are devoted to the application of Matlab in mathematical analysis, see the work of Stephen [22], Dunn [6], Shampine and Robert [16].

The distinct scientific communities that are working on various aspects of automatic analysis of data include Combinatorial Pattern Matching, Data Mining, Computational Statistics, Network Analysis, Text Mining, Image Processing, Syntactical Pattern Recognition, Machine Learning, Statistical Pattern Recognition, Computer Vision, and many others.

Bessel functions are a series of solutions to a second order differential equation that arise in many diverse situations. While special types of what would later be known as Bessel functions were studied by Euler, Lagrange, and the Bernoullis, the Bessel functions were first used by $\mathrm{F}$. W. Bessel to describe three body motion, with the Bessel functions appearing in the series expansion on planetary perturbation [8]. The subject of Bessel Functions and applications is a very rich subject; nevertheless, due to space and time restrictions and in the interest of studying applications. Appropriate development of zeroes, modified Bessel functions, and the application of boundary conditions can be studied in the work of Jennifer Niedziela [9].

In this paper, an attempt is made to introduce a new sequence of functions $\left\{V_{n}^{(v, \alpha)}(x ; a, k, s) / n=0,1,2, \ldots\right\}$, which involving the Bessel function of first kind $J_{v}(x)$. By using operational techniques, Some interesting generating relations and summation formulas are obtained. Program is made using the Matlab to establish the Database and Graph of the new sequence of function for different values of parameters.

The Bessel function of first kind is defined as [15, p.109]: 
$J_{v}(x)=\sum_{k=0}^{\infty} \frac{(-1)^{k}(x)^{v+2 k}}{2^{v+2 k} k ! \Gamma(v+k+1)}$

Operational techniques have drawn the attention of several researchers in the study of sequences of functions and polynomials.

In 1956, Chak [1] defined a class of polynomials as:

$$
G_{n, k}^{(\alpha)}(x)=x^{-\alpha-k n+n} e^{x}\left(x^{k} D\right)^{n}\left[x^{\alpha} e^{-x}\right]
$$

Where $k$ is constant and $n=0,1,2, \ldots, D \equiv \frac{d}{d x}$.

Gould and Hopper [7] introduced generalized Hermite polynomials in 1962 as:

$H_{n}^{r}(x, a, p)$

$=(-1)^{n} x^{-a} \exp \left(p x^{r}\right) D^{n}\left[x^{a} \exp \left(-p x^{r}\right)\right]$

Chatterjea [4] studied as a class of polynomials for generalized Laguerre polynomials in 1964:

$T_{r n}^{(\alpha)}(x, p)=$

$\frac{1}{n !} x^{-\alpha-n-1} \exp \left(p x^{r}\right)\left(x^{2} D\right)^{n}\left[x^{\alpha+1} \exp \left(-p x^{r}\right)\right]$

In 1968, Singh [17] studied the generalized Truesdell polynomials defined as:

$T_{n}^{(\alpha)}(x, r, p)$

$=x^{-\alpha} \exp \left(p x^{r}\right)(x D)^{n}\left[x^{\alpha} \exp \left(-p x^{r}\right)\right]$

Srivastava and Singh [19] introduced a general class of polynomials in 1971 as:

$G_{n}^{(\alpha)}(x, r, p, k)=$

$\frac{1}{n !} x^{-\alpha-k n} \exp \left(p x^{r}\right)\left(x^{k+1} D\right)^{n}\left[x^{\alpha} \exp \left(-p x^{r}\right)\right]$

In 1971, the Rodrigues formula for generalized Lagurre polynomials is given by Mittal [11] as:

$$
\begin{aligned}
& T_{k n}^{(\alpha)}(x) \\
& =\frac{1}{n !} x^{-\alpha} \exp \left(p_{k}(x)\right) D^{n}\left[x^{\alpha+n} \exp \left(-p_{k}(x)\right)\right]
\end{aligned}
$$

Where $p_{k}(x)$ is a polynomial in $x$ of degree $k$.

Mittal [12] also proved following relation for (1.7) as:

$$
\begin{aligned}
& T_{k n}^{(\alpha+s-1)}(x) \\
& =\frac{1}{n !} x^{-\alpha-n} \exp \left(p_{k}(x)\right) T_{s}^{n}\left[x^{\alpha} \exp \left(-p_{k}(x)\right)\right]
\end{aligned}
$$

Where $s$ is constant and $T_{s} \equiv x(s+x D)$.
Recently, Shukla and Prajapati [21] obtained several propertie of (7) .

Chandel [2] also studied a class of polynomials defined in 1973 as:

$$
\begin{aligned}
& T_{n}^{\alpha, k}(x, r, p) \\
& =x^{-\alpha} \exp \left(p x^{r}\right)\left(x^{k} D\right)^{n}\left[x^{\alpha} \exp \left(-p x^{r}\right)\right]
\end{aligned}
$$

In 1974, Chandel [3] established a generalization of polynomial system as:

$$
G_{n}(h, g(x), k)=e^{-h g(x)}\left(x^{k} D\right)^{n}\left[e^{-h g(x)}\right]
$$

Where $g(x)$ is a function of $x, h$ and $k$ are constants.

In the same year, Srivastava [20] discussed some operational formulas generalized function $F_{n}^{(r, m)}(x, a, k, p)$ in following the form:

$$
\begin{aligned}
& F_{n}^{(r, m)}(x, a, k, p) \\
& =x^{-a} \exp \left(p x^{r}\right)\left(x^{k} D\right)^{n}\left[x^{a+k m} \exp \left(-p x^{r}\right)\right]
\end{aligned}
$$

In 1975, Joshi and Parjapat [10] introduced the a class of polynomial:

$$
\begin{aligned}
& M_{v n}^{(\alpha)}(x, a, k) \\
& =\frac{x^{-\alpha-n k}}{n !} \exp \left(p_{v}(x)\right)\left[x^{k}(a+x D)\right]^{n} \\
& {\left[x^{\alpha} \exp \left(-p_{v}(x)\right)\right]}
\end{aligned}
$$

Subsequently in 1975, Patil and Thakare [14] have obtained several formulae and generating relation for:

$$
\begin{aligned}
& P_{n}^{(\alpha)}(x, r, s, a, k, \lambda) \\
& =x^{-\alpha} \exp \left(p x^{r}\right)\left[x^{k}(\lambda+x D)\right]^{n}\left[x^{\alpha+s n} \exp \left(-p x^{r}\right)\right]^{(}
\end{aligned}
$$

In 1979, Srivastava and Singh [18] studied a sequence of functions $V_{n}^{(\alpha)}(x ; a, k, s)$ defined as:

$$
\begin{aligned}
& V_{n}^{(\alpha)}(x ; a, k, s) \\
& =\frac{x^{-\alpha}}{n !} \exp \left\{p_{k}(x)\right\} \theta^{n}\left[x^{\alpha} \exp \left\{-p_{k}(x)\right\}\right]
\end{aligned}
$$

By employing the operator $\theta \equiv x^{a}(s+x D)$, where $s$ is constant and $p_{k}(x)$ is a polynomial in $x$ of degree $k$

A new sequence of function $\left\{V_{n}^{(v, \alpha)}(x ; a, k, s) / n=0,1,2, \ldots\right\}$ is introduced in this paper as:

$$
\begin{aligned}
& V_{n}^{(v, \alpha)}(x ; a, k, s) \\
& =\frac{1}{n !} x^{-\alpha} J_{v}\left(p_{k}(x)\right)\left(T_{x}^{a, s}\right)^{n}\left[x^{\alpha} J_{v}\left(-p_{k}(x)\right)\right]
\end{aligned}
$$


Where $T_{x}^{a, s} \equiv x^{a}(s+x D), D \equiv \frac{d}{d x}, a$ and $s$ are constants, $\beta \geq 0, k$ is finite and non-negative integer, $p_{k}(x)$ is a polynomial in $x$ of degree $k$ and $J_{v}(x)$ is a Bessel function of first kind defined in equation (1.1).

Some generating relations and finite summation formulae of class of polynomials or sequence of function have been obtained by using the properties of the differential operators. $T_{x}^{a, s} \equiv x^{a}(s+x D), T_{x}^{a, 1} \equiv x^{a}(1+x D)$, where $D \equiv \frac{d}{d x}$, is based on the work of Mittal [13], Patil and Thakare [14], Srivastava and Singh[18].

Some useful Operational Techniques are given below:

$$
\begin{aligned}
& \exp \left(t T_{x}^{a, s}\right)\left(x^{\beta} f(x)\right) \\
& =x^{\beta}\left(1-a x^{a} t\right)^{-\left(\frac{\beta+s}{a}\right)} f\left(x\left(1-a x^{a} t\right)^{-1 / a}\right) \\
& \exp \left(t T_{x}^{a, s}\right)\left(x^{\alpha-a n} f(x)\right) \\
& =x^{\alpha}(1+a t)^{-1+\left(\frac{\alpha+s}{a}\right)} f\left(x(1+a t)^{1 / a}\right) \\
& \left(T_{x}^{a, s}\right)^{n}(x u v) \\
& =x \sum_{m=0}^{\infty}\left(\begin{array}{l}
n \\
m
\end{array}\right)\left(T_{x}^{a, s}\right)^{n-m}(v)\left(T_{x}^{a, 1}\right)^{m}(u) \\
& (1+x D)(1+a+x D)(1+2 a+x D) \\
& (1+3 a+x D)^{\prime . .(1+(m-1) a+x D) x^{\beta-1}} \\
& =a^{m}\left(\frac{\beta}{a}\right)_{m} x^{\beta-1} \\
& (1-a t)^{\frac{-\alpha}{a}}=(1-a t)^{\frac{-\beta}{a}} \sum_{m=0}^{\infty}\left(\frac{\alpha-\beta}{a}\right)_{m} \frac{(a t)^{m}}{m !}
\end{aligned}
$$

\section{GENERATING RELATIONS}

$\sum_{n=0}^{\infty} V_{n}^{(v, \alpha)}(x ; a, k, s) x^{-a n} t^{n}$

$=(1-a t)^{-\left(\frac{\alpha+s}{a}\right)} J_{v}\left(p_{k}(x)\right)$

$J_{v}\left(-p_{k}\left(x(1-a t)^{-1 / a}\right)\right)$

$\sum_{n=0}^{\infty} V_{n}^{(v, \alpha-a n)}(x ; a, k, s) x^{-a n} t^{n}$

$=(1+a t)^{-1+\left(\frac{\alpha+s}{a}\right)} J_{v}\left(p_{k}(x)\right)$

$J_{v}\left(-p_{k}\left(x(1+a t)^{1 / a}\right)\right)$ $\sum_{n=0}^{\infty}\left(\begin{array}{c}m+n \\ m\end{array}\right) V_{n}^{(v, \alpha)}(x ; a, k, s) t^{n}$

$=(1-a t)^{-\left(\frac{\alpha+s}{a}\right)} \frac{J_{v}\left(p_{k}(x)\right)}{J_{v}\left(p_{k}\left(x(1-a t)^{-1 / a}\right)\right)}$

$V_{n}^{(v, \alpha)}\left(x(1-a t)^{-1 / a} ; a, k, s\right)$

Proof of (2.1):

From (1.15), we consider

$\sum_{n=0}^{\infty} V_{n}^{(v, \alpha)}(x ; a, k, s) t^{n}=$

$x^{-\alpha} J_{v}\left(p_{k}(x)\right) \exp \left(t T_{x}^{a, s}\right)\left[x^{\alpha} J_{v}\left(-p_{k}(x)\right)\right]$

Using operational technique (1.16), above equation (2.4) reduces to:

$$
\begin{aligned}
& \sum_{n=0}^{\infty} V_{n}^{(v, \alpha)}(x ; a, k, s) t^{n}= \\
& x^{-\alpha} J_{v}\left(p_{k}(x)\right) x^{\alpha}\left(1-a x^{a} t\right)^{-\left(\frac{\alpha+s}{a}\right)} J_{v}\left(-p_{k}\left(x\left(1-a x^{a} t\right)^{-1 / a}\right)\right) \\
& =\left(1-a x^{a} t\right)^{-\left(\frac{\alpha+s}{a}\right)} J_{v}\left(p_{k}(x)\right) J_{v}\left(-p_{k}\left(x\left(1-a x^{a} t\right)^{-1 / a}\right)\right)
\end{aligned}
$$

And replacing $t$ by $t x^{-a}$, this gives (2.1).

Proof of (23):

Again from (1.15), we have

$$
\begin{aligned}
& \sum_{n=0}^{\infty} x^{-a n} V_{n}^{(v, \alpha-a n)}(x ; a, k, s) t^{n} \\
& =x^{-\alpha} J_{v}\left(p_{k}(x)\right) \exp \left(t T_{x}^{a, s}\right)\left[x^{\alpha-a n} J_{v}\left(-p_{k}(x)\right)\right]
\end{aligned}
$$

applying the operational technique (1.17), we get

$$
\begin{aligned}
& \sum_{n=0}^{\infty} x^{-a n} V_{n}^{(v, \alpha-a n)}(x ; a, k, s) t^{n} \\
& =x^{-\alpha} J_{v}\left(p_{k}(x)\right) x^{\alpha}(1+a t)^{\frac{\alpha+s}{a}-1} J_{v}\left[-p_{k}\left(x(1+a t)^{1 / a}\right)\right] \\
& =(1+a t)^{\frac{\alpha+s}{a}-1} J_{v}\left(p_{k}(x)\right) J_{v}\left[-p_{k}\left(x(1+a t)^{1 / a}\right)\right]
\end{aligned}
$$

This proves (2.2).

\section{Proof of (2.3):}

We can write (1.15) as,

$$
\begin{aligned}
& \left(T_{x}^{a, s}\right)^{n}\left[x^{\alpha} J_{v}\left(-p_{k}(x)\right)\right] \\
& =n ! x^{\alpha} \frac{1}{J\left(p_{k}(x)\right)} V_{n}^{(v, \alpha)}(x ; a, k, s)
\end{aligned}
$$

Or 
$\exp \left(t\left(T_{x}^{a, s}\right)\right)\left\{\left(T_{x}^{a, s}\right)^{n}\left[x^{\alpha} J_{v}\left(-p_{k}(x)\right)\right]\right\}$

$=n ! \exp \left(t T_{x}^{a, \alpha}\right)\left[x^{\alpha} \frac{1}{J\left(p_{k}(x)\right)} V_{n}^{(v, \alpha)}(x ; a, k, s)\right]$

$\sum_{m=0}^{\infty} \frac{t^{m}}{m !}\left(T_{x}^{a, s}\right)^{m+n}\left[x^{\alpha} J_{v}\left(-p_{k}(x)\right)\right]$

$=n ! \exp \left(T_{x}^{a, s}\right)\left[x^{\alpha} \frac{1}{J\left(p_{k}(x)\right)} V_{n}^{(v, \alpha)}(x ; a, k, s)\right]$

Using the operational technique (1.16), above equation can be written as

$\sum_{m=0}^{\infty} \frac{t^{m}}{m !}\left(T_{x}^{a, s}\right)^{m+n}\left[x^{\alpha} J_{v}\left(-p_{k}(x)\right)\right]$

$=n ! x^{\alpha}\left(1-a x^{a} t\right)^{-\left(\frac{\alpha+s}{a}\right)} \frac{1}{J_{v}\left(p_{k}\left(x\left(1-a x^{a} t\right)^{-1 / a}\right)\right)}$

$V_{n}^{(v, \alpha)}\left(x\left(1-a x^{a} t\right)^{-1 / a} ; a, k, s\right)$

use of (2.8) gives

$\sum_{m=0}^{\infty} \frac{t^{m}(m+n) !}{m ! n !} x^{\alpha} \frac{1}{J_{v}\left(p_{k}(x)\right)} V_{m+n}^{(v, \alpha)}(x ; a, k, s)$

$=x^{\alpha}\left(1-a x^{a} t\right)^{-\left(\frac{\alpha+s}{a}\right)} \frac{V_{n}^{(v, \alpha)}\left(x\left(1-a x^{a} t\right)^{-1 / a} ; a, k, s\right)}{J_{v}\left[p_{k}\left(x\left(1-a x^{a} t\right)^{-1 / a}\right)\right]}$

Therefore

$\sum_{m=0}^{\infty}\left(\begin{array}{c}m+n \\ n\end{array}\right) V_{m+n}^{(v, \alpha)}(x ; a, k, s) t^{m}$

$=\left(1-a x^{a} t\right)^{-\left(\frac{\alpha+s}{a}\right)} \frac{J_{v}\left(p_{k}(x)\right)}{J_{v}\left(p_{k}\left(x\left(1-a x^{a} t\right)^{-1 / a}\right)\right)}$

$V_{n}^{(v, \alpha)}\left(x\left(1-a x^{a} t\right)^{-1 / a} ; a, k, s\right)$

And replacing $t$ by $t x^{-a}$, this gives the result (2.3).

\section{FINITE SUMMATION FORMULAE}

$V_{n}^{(v, \alpha)}(x ; a, k, s)$

$=\sum_{m=0}^{\infty} \frac{1}{m !}\left(a x^{a}\right)^{m}\left(\frac{\alpha}{a}\right)_{m} V_{n-m}^{(v, 0)}(x ; a, k, s)$

$V_{n}^{(v, \alpha)}(x ; a, k, s)$

$=\sum_{m=0}^{\infty} \frac{1}{m !}\left(a x^{a}\right)^{m}\left(\frac{\alpha-\beta}{a}\right)_{m} V_{n-m}^{(v, \beta)}(x ; a, k, s)$

\section{Proof of the (3.1) equation:}

From equation (1.15), We have:

$V_{n}^{(v, \alpha)}(x ; a, k, s)$

$=\frac{1}{n !} x^{-\alpha} J_{v}\left(p_{k}(x)\right)\left(T_{x}^{(a, s)}\right)^{n}\left[x x^{\alpha-1} J_{v}\left(-p_{k}(x)\right)\right]$
Using the operational technique (1.18), we have:

$V_{n}^{(v, \alpha)}(x ; a, k, s)$

$=\frac{1}{n !} x^{-\alpha} J_{v}\left(p_{k}(x)\right) x \sum_{m=0}^{n}\left(\begin{array}{l}n \\ m\end{array}\right)\left(T_{x}^{(a, s)}\right)^{n-m}\left[J_{v}\left(-p_{k}(x)\right)\right]\left(T_{x}^{a, 1}\right)^{m}\left(x^{\alpha-1}\right)$

$=\frac{1}{n !} x^{-\alpha} J_{v}\left(p_{k}(x)\right) x \sum_{m=0}^{n} \frac{n !}{m !(n-m) !} x^{a(n-m)} \times$

$[(s+x D)(s+a+x D)(s+2 a+x D) \ldots$

$(s+(n-m-1) a+x D)]\left[J_{v}\left(-p_{k}(x)\right)\right] x^{a m} \times$

$[(1+x D)(1+a+x D)(1+2 a+x D) .$.

$(1+(m-1) a+x D)]\left(x^{\alpha-1}\right)$

$=J_{v}\left(p_{k}(x)\right) \sum_{m=0}^{n} \frac{1}{m !(n-m) !} x^{a n}$

$\prod_{i=0}^{n-m-1}(s+i a+x D)\left[J_{v}\left(-p_{k}(x)\right)\right] a^{m}\left(\frac{\alpha}{a}\right)_{m}$

Put $\alpha=0$ and replacing $n$ by $n-m$ in (3.3), we get: $V_{n-m}^{(v, 0)}(x ; a, k, s)$

$=\frac{1}{(n-m) !} J_{v}\left(p_{k}(x)\right)\left(T_{x}^{a, s}\right)^{n-m}\left[J_{v}\left(-p_{k}(x)\right)\right]$

$\Rightarrow \frac{1}{(n-m) !}\left(T_{x}^{a, s}\right)^{n-m}\left[J_{v}\left(-p_{k}(x)\right)\right]$

$=\frac{1}{J_{v}\left(p_{k}(x)\right)} V_{n-m}^{(v, 0)}(x ; a, k, s)$

This gives

$\frac{1}{(n-m) !} \prod_{i=0}^{n-m-1}(s+i a+x D)\left[J_{v}\left(-p_{k}(x)\right)\right]$

$=x^{a(m-n)} \frac{1}{J_{v}\left(p_{k}(x)\right)} V_{n-m}^{(v, 0)}(x ; a, k, s)$

From equation (3.4) and (3.6) we have the main the result

Proof the equation (3.2):

Equation (1.15) can be written as:

$\sum_{n=0}^{\infty} V_{n}^{(v, \alpha)}(x ; a, k, s) t^{n}$

$=x^{-\alpha} J_{v}\left(p_{k}(x)\right) \exp \left(T_{x}^{(a, s)}\right)\left[x^{\alpha} J_{v}\left(-p_{k}(x)\right)\right]$

Applying the (1.16) in equation (3.1), we have:

$\sum_{n=0}^{\infty} V_{n}^{(v, \alpha)}(x ; a, k, s) t^{n}$

$=x^{-\alpha} J_{v}\left(p_{k}(x)\right) x^{\alpha}\left(1-a x^{a} t\right)^{-\left(\frac{\alpha+s}{a}\right)} J_{v}\left(-p_{k}\left(x\left(1-a x^{a} t\right)^{-1 / a}\right)\right)$

$=\left(1-a x^{a} t\right)^{-\left(\frac{\alpha+s}{a}\right)} J_{v}\left(p_{k}(x)\right) J_{v}\left(-p_{k}\left(x\left(1-a x^{a} t\right)^{-1 / a}\right)\right)$ 
Applying the result from equation (1.20); equation (3.8) reduces to:

$$
\begin{aligned}
& =\left(1-a x^{a} t\right)^{-\left(\frac{\beta+s}{a}\right)} \sum_{m=0}^{\infty}\left(\frac{\alpha-\beta}{a}\right)_{m} \frac{\left(a x^{a} t\right)^{m}}{m !} \\
& J_{v}\left(p_{k}(x)\right) J_{v}\left(-p_{k}\left(x\left(1-a x^{a} t\right)^{-1 / a}\right)\right) \\
& =\sum_{m=0}^{\infty}\left(\frac{\alpha-\beta}{a}\right)_{m} \frac{\left(a x^{a} t\right)^{m}}{m !} x^{-\beta} J_{v}\left(p_{k}(x)\right) \\
& \exp \left(T_{x}^{a, s}\right)\left[x^{\beta} J_{v}\left(-p_{k}(x)\right)\right] \\
& =\sum_{m=0}^{\infty} \sum_{n=0}^{\infty}\left(\frac{\alpha-\beta}{a}\right)_{m} \frac{\left(a x^{a}\right)^{m} t^{n+m}}{m ! n !} x^{-\beta} \\
& J_{v}\left(p_{k}(x)\right)\left(T_{x}^{a, s}\right)^{n}\left[x^{\beta} J_{v}\left(-p_{k}(x)\right)\right] \\
& =\sum_{n=0}^{\infty} \sum_{m=0}^{n}\left(\frac{\alpha-\beta}{a}\right)_{m} \frac{\left(a x^{a}\right)^{m} t^{n}}{m !(n-m) !} x^{-\beta} \\
& J_{v}\left(p_{k}(x)\right)\left(T_{x}^{a, s}\right)^{n-m}\left[x^{\beta} J_{v}\left(-p_{k}(x)\right)\right]
\end{aligned}
$$

Now equating the coefficient of $t^{n}$, we get

$V_{n}^{(v, \alpha)}(x ; a, k, s)$

$=\sum_{m=0}^{n}\left(\frac{\alpha-\beta}{a}\right)_{m} \frac{\left(a x^{a}\right)^{m}}{m !(n-m) !} x^{-\beta}$

$$
J_{v}\left(p_{k}(x)\right)\left(T_{x}^{a, s}\right)^{n-m}\left[x^{\beta} J_{v}\left(-p_{k}(x)\right)\right]
$$

Using the equation (1.15) in (3.10) we have the result (3.2)

\section{SPECIAL CASES}

The interesting special cases/relationships between (1.15) and class of polynomials (1.2)-(1.14) can also be obtained for appropriate values of alpha, nu, $a, k$ and $s$.

The, Matlab is one of the important aspect mainly in the field of sciences and engineering, Therefore, the imperative Matlab coding established for each parameter of equation (1.15) and some interesting database and graphs also established in the section 5. Using this coding reader may obtain large number of graphs of equation (1.15), which gives the eccentric characteristics in the area of sequence of functions or class of polynomials

\section{PROGRAMMING OF THE NEW SEQUENCE OF FUNCTIONS IN MATLAB}

Code of New sequence of functions:

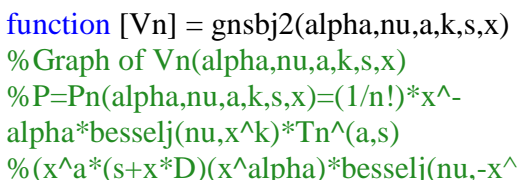

$\%\left(x^{\wedge} a *\left(s+x^{*} D\right)\left(x^{\wedge}\right.\right.$ alpha $) *$ besselj $\left.\left(n u,-x^{\wedge} k\right)\right)$, where $n=1,2,3, \ldots$ syms $x$

$\%$ n=input('please enter $n: ')$;

$\mathrm{n}=2$;

$\mathrm{E} 1=\operatorname{besselj}\left(\mathrm{nu},-\mathrm{x} \cdot{ }^{\wedge} \mathrm{k}\right)$

$\mathrm{y}=\left(\mathrm{x} .{ }^{\wedge}\right.$ alpha $) \cdot{ }^{*} \mathrm{E} 1$;

for $\mathrm{i}=1: \mathrm{n}$

$\mathrm{y}=\left(\mathrm{x} \cdot{ }^{\wedge} \mathrm{a}\right) \cdot *\left(\mathrm{~s} \cdot{ }^{*} \mathrm{y}+\mathrm{x} . *^{*} \operatorname{diff}(\mathrm{y})\right)$

end

$\mathrm{E} 2=$ besselj(nu,x.^k);

$\mathrm{v}=(1 . /$ factorial(n) $) . *\left(1 . /\left(\mathrm{x} .{ }^{\wedge}\right.\right.$ alpha $\left.)\right) . * \mathrm{E} 2 . * \mathrm{y}$;

$\% \mathrm{Vn}=\operatorname{subs}(\mathrm{v}, \mathrm{x},-.5: .1: .5)$;

$\mathrm{Vn}=\operatorname{subs}(\mathrm{v}, \mathrm{x})$;

end

Plot The Graph:

Command window:

hold on

$\mathrm{V} 1=\operatorname{ezplot}(\operatorname{gnsbj} 1(1,1,1,1,1, \mathrm{x}),[-2: .5: 2])$;

$\mathrm{V} 2=\operatorname{ezplot}(\operatorname{gnsbj} 2(1,1,1,1,1, \mathrm{x}),[-2: .5: 2])$;

$\mathrm{V} 3=\operatorname{ezplot}(\operatorname{gnsbj} 3(1,1,1,1,1, \mathrm{x}),[-2: .5: 2])$;

$\mathrm{V} 4=\operatorname{ezplot}(\operatorname{gnsbj} 4(1,1,1,1,1, \mathrm{x}),[-2: .5: 2])$;

title('Vn(alpha,nu,a,k,s,x);n=1,2,3,4')

hold off

set(V1,'color','r')

$\operatorname{set}(\mathrm{V} 2$, 'color','b')

set(V3,'color','g')

set(V4,'color','m')

legend('V1(1,1,1,1,1,x)','V2(1,1,1,1,1,x)','V3(1,1,1,1,1,x)','V4( $\left.1,1,1,1,1, \mathrm{x})^{\prime}\right)$

\section{DIFFERENT DATABASES AND GRAPHS USING MATLAB}

The new sequence $\left\{V_{n}^{(v, \alpha)}(x ; a, k, s) / n=0,1,2, \ldots\right\}$ introduced in equation (1.15), takes place in the form of $\mathrm{Vn}$ (alpha,nu,a, $\mathrm{k}, \mathrm{s}, \mathrm{x})$ to establish Database and Graph for different values of parameters alpha, nu, a, $\mathrm{k}, \mathrm{s}$ and $(\mathrm{n}=0,1,2,3, \ldots)$. Four different Database for different values of parameters for $\mathrm{n}=1,2,3$ in the interval $-2 \leq x \leq 2$ with difference .5 and $-0.5 \leq x \leq 0.5$ with difference 0.1 are established, as shown in Data base in table 1, 2, 3, 4 \& 5 and their corresponding Graphs are plotted.

\section{(6.1) First Database and Graph:}

Table 1. Database of sequence of function $\operatorname{Vn}(1,1,1,1,1, \mathrm{x})$ for $\mathrm{n}=1: 4$

\begin{tabular}{|c|c|c|c|c|}
\hline \multicolumn{5}{|c|}{ Vn(alpha,nu,a,k,s,x); alpha=1;nu=1; $\mathrm{a}=1 ; \mathrm{k}=1 ; \mathrm{s}=1 ; \mathrm{n}=1: 4$} \\
\hline $\mathrm{x}$ & $\mathrm{V} 1(1,1,1,1,1, \mathrm{x})$ & $\mathrm{V} 2(1,1,1,1,1, \mathrm{x})$ & $\mathrm{V} 3(1,1,1,1,1, \mathrm{x})$ & $\mathrm{V} 4(1,1,1,1,1, \mathrm{x})$ \\
\hline-2.0 & 1.1817 & -1.2520 & -7.1588 & 51.7684 \\
\hline-1.5 & 1.1095 & -2.3219 & 3.0743 & -0.5750 \\
\hline
\end{tabular}




\begin{tabular}{|c|c|c|c|c|}
\hline-1.0 & 0.5304 & -0.9386 & 1.3300 & -1.6043 \\
\hline-0.5 & 0.0862 & -0.0839 & 0.0676 & -0.0488 \\
\hline 0 & 0 & 0 & 0 & 0 \\
\hline 0.5 & -0.0862 & -0.0839 & -0.0676 & -0.0488 \\
\hline 1.0 & -0.5304 & -0.9386 & -1.3300 & -1.6043 \\
\hline 1.5 & -1.1095 & -2.3219 & -3.0743 & -0.5750 \\
\hline 2.0 & -1.1817 & -1.2520 & 7.1588 & 51.7684 \\
\hline
\end{tabular}

\section{Vn(alpha,nu,a,k,s,x);n=1,2,3,4}

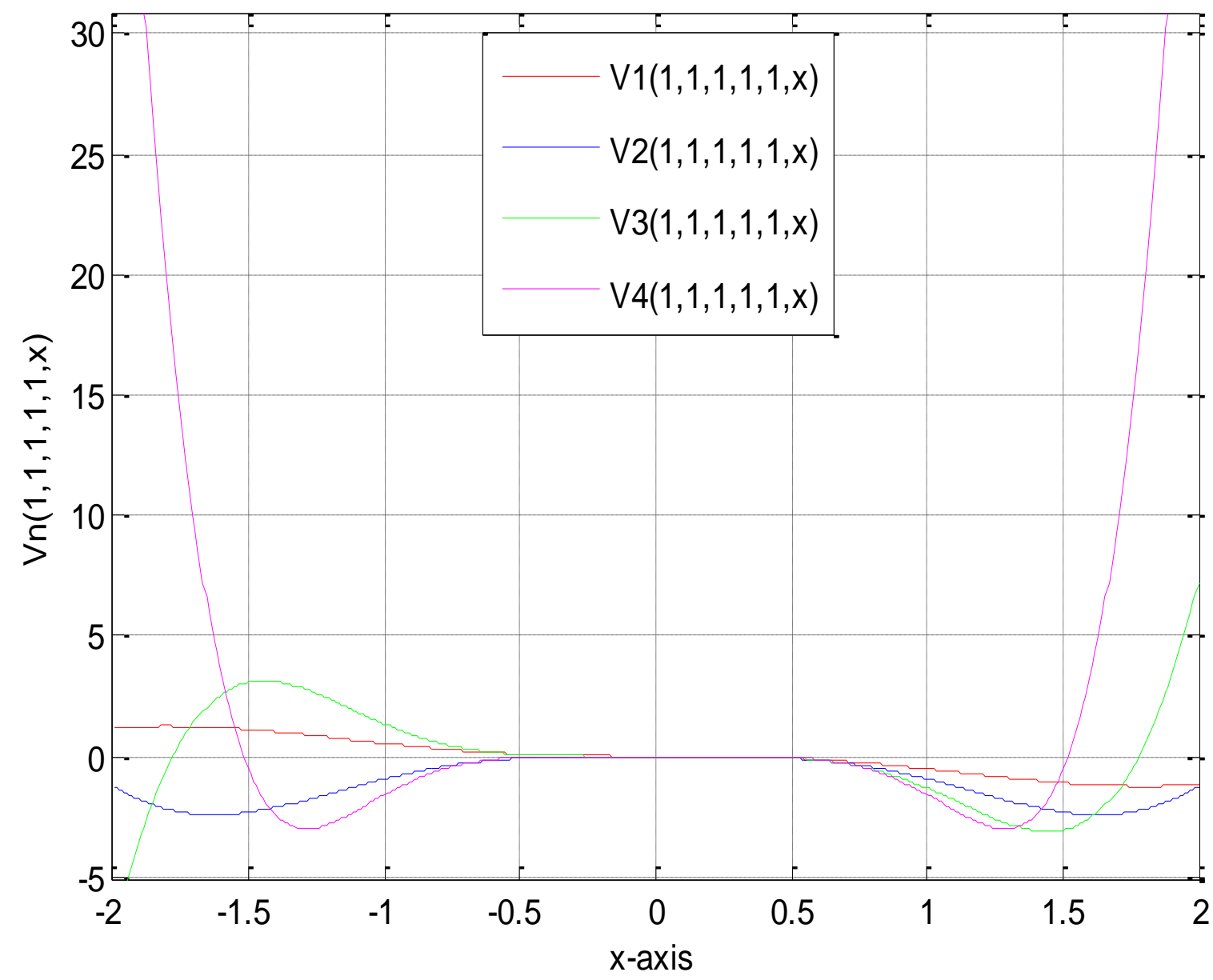

\section{(6.2) Second Database and Graph:}

Fig. 1 Based on table (1)

Table 2. Database of sequence of function $\operatorname{Vn}(-1,2,1,2,1)$ for $\mathrm{n}=1: 4$

\begin{tabular}{|c|c|c|c|c|}
\hline \multicolumn{5}{|c|}{ Vn(alpha,nu,a,k,s,x); alpha=-1;nu=2; a =1; $\mathrm{k}=2 ; \mathrm{s}=1 ; \mathrm{n}=1: 4$} \\
\hline $\mathrm{x}$ & $\mathrm{V} 1(-1,2,1,2,1, \mathrm{x})$ & $\mathrm{V} 2(-1,2,1,2,1, \mathrm{x})$ & $\mathrm{V} 3(-1,2,1,2,1, \mathrm{x})$ & $\mathrm{V} 4(-1,2,1,2,1, \mathrm{x})$ \\
\hline-2.0 & 1.4455 & -14.1741 & 26.3861 & 313.2720 \\
\hline-1.5 & -0.5153 & -0.3966 & 9.0717 & -42.1274 \\
\hline-1.0 & -0.0483 & 0.1034 & -0.1567 & 0.1564 \\
\hline-0.5 & -0.0001 & 0.0001 & -0.0001 & 0.0001 \\
\hline 0 & 0 & 0 & 0 & 0 \\
\hline
\end{tabular}




\begin{tabular}{|l|l|l|l|l|}
\hline 0.5 & 0.0001 & 0.0001 & 0.0001 & 0.0001 \\
\hline 1.0 & 0.0483 & 0.1034 & 0.1567 & 0.1564 \\
\hline 1.5 & 0.5153 & -0.3966 & -9.0717 & -42.1274 \\
\hline 2.0 & -1.4455 & -14.1741 & -26.3861 & 313.2720 \\
\hline
\end{tabular}

Vn(alpha, nu,a,k,s,x);n=1,2,3,4

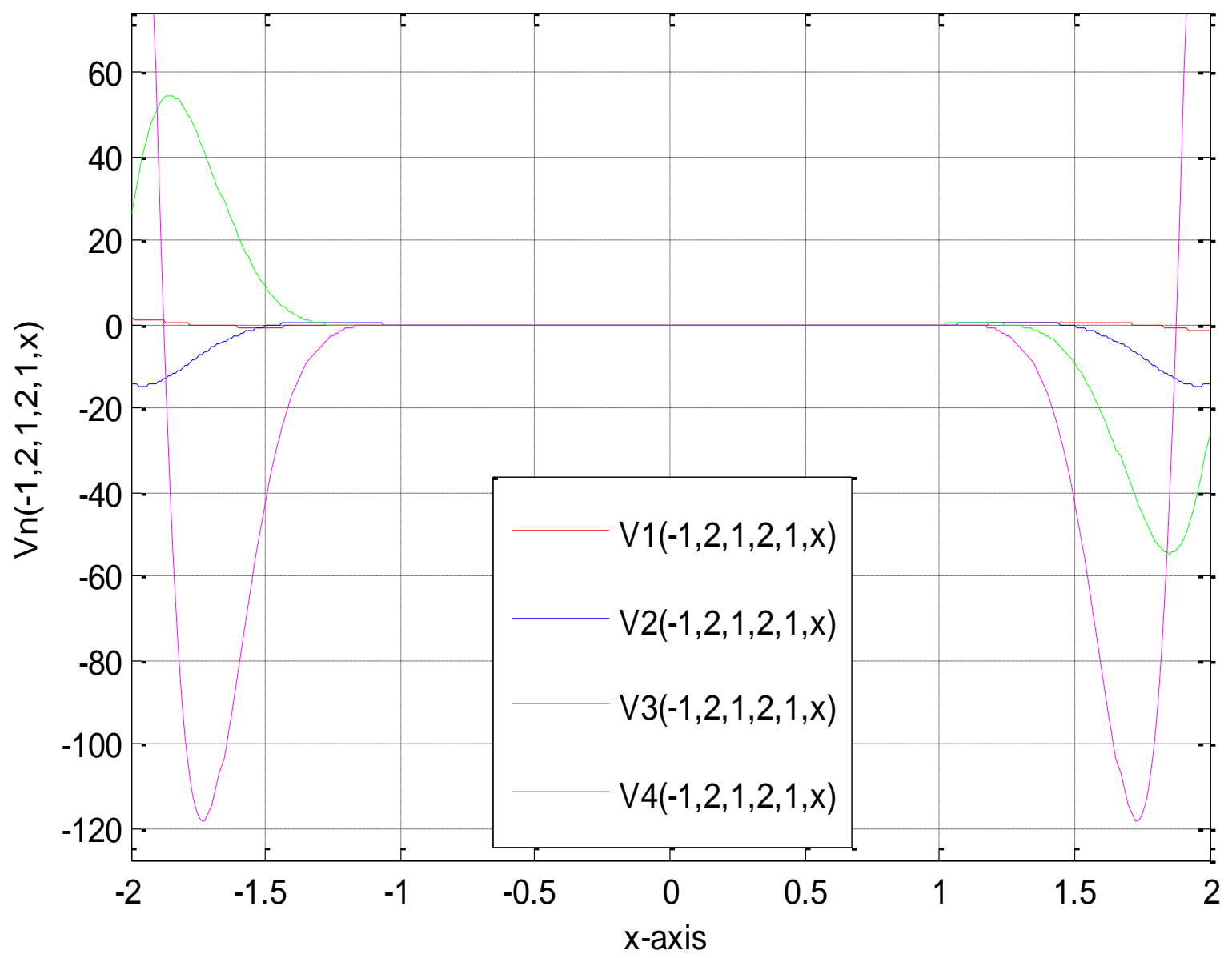

Fig. 2 Based on table (2)

\section{(6.3) Third Database and Graph:}

Table 3. Database of sequence of function V1 for different values of parameters Vn(alpha,nu,a,k,s,x); alpha=2,4,6;nu=2; a=3; k=2; s=1;n=1

\begin{tabular}{|c|c|c|c|c|}
\hline \multicolumn{5}{|c|}{ Vn(alpha,nu,a,k,s,x); alpha=2,4,6;nu=2; a=3; $=2 ; \mathrm{s}=1 ; \mathrm{n}=1$} \\
\hline $\mathrm{x}$ & $\mathrm{V} 1(2,2,3,2,1, \mathrm{x})$ & $\mathrm{V} 1(4,2,3,2,1, \mathrm{x})$ & $\mathrm{V} 1(6,2,3,2,1, \mathrm{x})$ & $\mathrm{V} 1(8,2,3,2,1, \mathrm{x})$ \\
\hline-2.0 & 2.5998 & 0.4784 & -1.6431 & -3.7645 \\
\hline-1.5 & -2.8178 & -3.9233 & -5.0288 & -0.1671 \\
\hline-1.0 & -0.0879 & -0.1143 & -0.1407 & -0.0001 \\
\hline-0.5 & -0.0001 & -0.0001 & -0.0001 & 0 \\
\hline 0 & 0 & 0 & 0 & 0.0001 \\
\hline 0.5 & 0.0001 & 0.0001 & 0.0001 & 0.1671 \\
\hline 1.0 & 0.0879 & 0.1143 & 0.1407 & 6.1343 \\
\hline 1.5 & 2.8178 & 3.9233 & 5.0288 & 3.7645 \\
\hline 2.0 & -2.5998 & -0.4784 & 1.6431 & \\
\hline
\end{tabular}




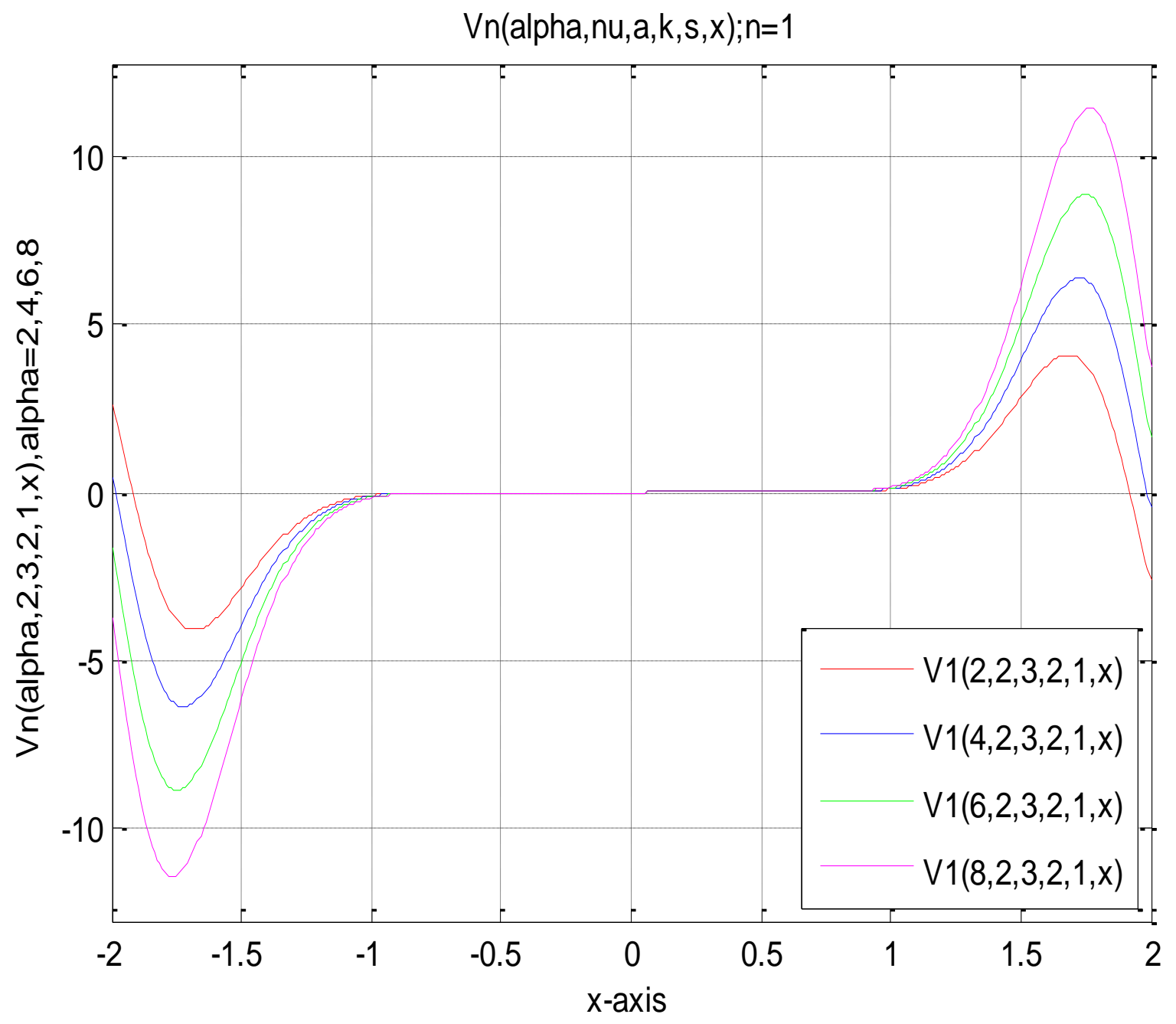

Fig. 3 Based on table (3)

\section{(6.4) Fourth Database and Graph:}

Table 4. Database of sequence of function V2 for different values of parameters

\begin{tabular}{|c|c|c|c|c|}
\hline \multicolumn{5}{|c|}{ Vn(alpha,nu,a,k,s,x); alpha=2,4,6,8;nu=2; a=3; $\mathrm{k}=2 ; \mathrm{s}=1 ; \mathrm{n}=2$} \\
\hline $\mathrm{x}$ & $\mathrm{V} 2(2,2,3,2,1, \mathrm{x})$ & $\mathrm{V} 2(4,2,3,2,1, \mathrm{x})$ & $\mathrm{V} 2(6,2,3,2,1, \mathrm{x})$ & $\mathrm{V} 2(8,2,3,2,1, \mathrm{x})$ \\
\hline-2.0 & -335.4357 & -334.6040 & -299.8294 & -231.1119 \\
\hline-1.5 & 30.4355 & 58.7832 & 94.5931 & 137.8653 \\
\hline-1.0 & 0.4155 & 0.6573 & 0.9520 & 1.2995 \\
\hline-0.5 & 0.0000 & 0.0001 & 0.0001 & 0.0001 \\
\hline 0 & 0 & 0 & 0 & 0 \\
\hline 0.5 & 0.0000 & 0.0001 & 0.0001 & 1.2995 \\
\hline 1.0 & 0.4155 & 0.6573 & 0.9520 & 137.8653 \\
\hline 1.5 & 30.4355 & 58.7832 & 94.5931 & -231.1119 \\
\hline 2.0 & -335.4357 & -334.6040 & -299.8294 & 0.001 \\
\hline
\end{tabular}




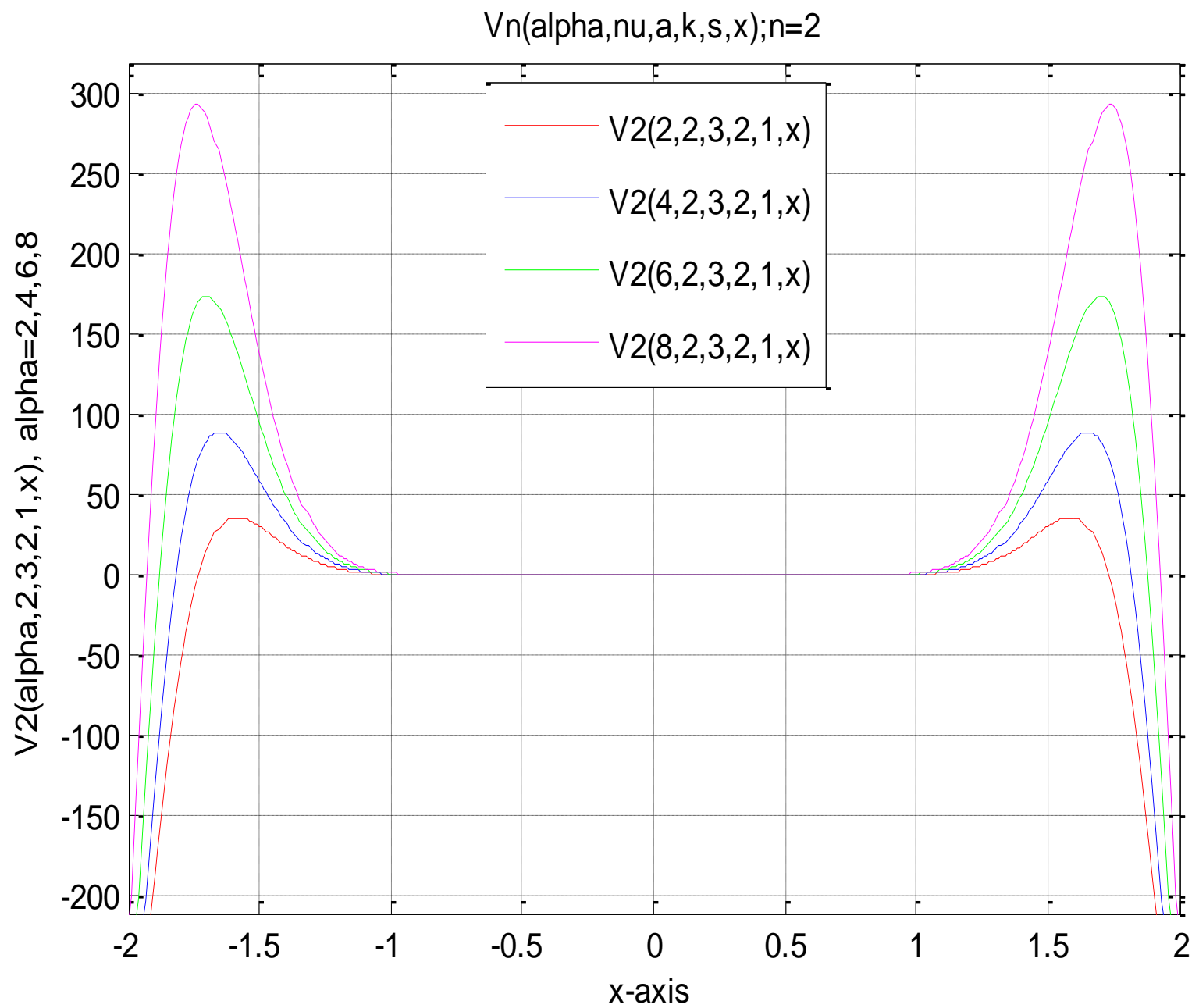

(6.5) Fifth Database and Graph:

Fig. 4 Based on table (4)

Table 5. Database of sequence of function V2 for different values of parameters

\begin{tabular}{|c|c|c|c|c|}
\hline \multicolumn{5}{|c|}{ Vn(alpha,nu,a,k,s,x); alpha=nu=1; a=2:5; $\mathrm{k}=3: 6 ; \mathrm{s}=4: 7 ; \mathrm{n}=2$} \\
\hline $\mathrm{x}$ & $\mathrm{V} 2(1,1,2,3,4, \mathrm{x})$ & $\mathrm{V} 2(1,1,3,4,5, \mathrm{x})$ & $\mathrm{V} 2(1,1,4,5,6, \mathrm{x})$ & $\mathrm{V} 2(1,1,5,6,7, \mathrm{x})$ \\
\hline-0.5 & -0.0097 & $-0.9899 \times 10^{-3}$ & $-0.9151 \times 10^{-4}$ & $-0.7926 \times 10^{-5}$ \\
\hline-0.4 & -0.0010 & $-0.0436 \times 10^{-3}$ & $-0.0165 \times 10^{-4}$ & $-0.0058 \times 10^{-5}$ \\
\hline-0.3 & -0.0001 & $-0.0008 \times 10^{-3}$ & $-0.0001 \times 10^{-4}$ & $-0.0000 \times 10^{-5}$ \\
\hline-0.2 & -0.0000 & $-0.0000 \times 10^{-3}$ & $-0.0000 \times 10^{-4}$ & $-0.0000 \times 10^{-5}$ \\
\hline-0.1 & -0.0000 & $-0.0000 \times 10^{-3}$ & $-0.0000 \times 10^{-4}$ & $-0.0000 \times 10^{-5}$ \\
\hline 0 & 0 & 0 & 0 & 0 \\
\hline 0.1 & -0.0000 & $-0.0000 \times 10^{-3}$ & $-0.0000 \times 10^{-4}$ & $-0.0000 \times 10^{-5}$ \\
\hline 0.2 & -0.0000 & $-0.0000 \times 10^{-3}$ & $-0.0000 \times 10^{-4}$ & $-0.0000 \times 10^{-5}$ \\
\hline 0.3 & -0.0001 & $-0.0008 \times 10^{-3}$ & $-0.0001 \times 10^{-4}$ & $-0.0000 \times 10^{-5}$ \\
\hline 0.4 & -0.0010 & $-0.0436 \times 10^{-3}$ & $-0.0165 \times 10^{-4}$ & $-0.0058 \times 10^{-5}$ \\
\hline 0.5 & -0.0097 & $-0.9899 \times 10^{-3}$ & $-0.9151 \times 10^{-4}$ & $-0.7926 \times 10^{-5}$ \\
\hline
\end{tabular}




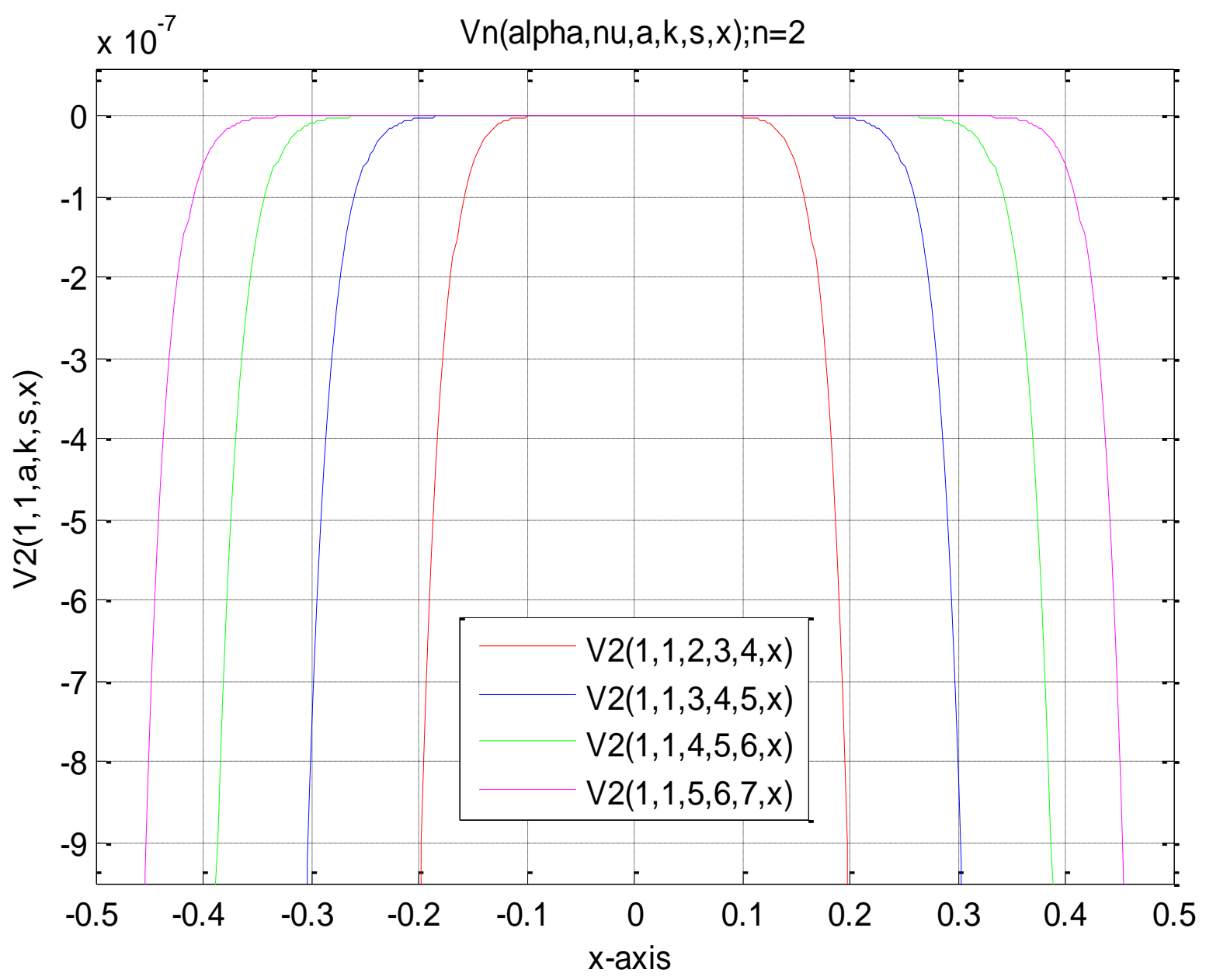

Fig. 5 Based on table (5)

(6.6) Different Graphs Of The Sequence Of Functions For Different Range

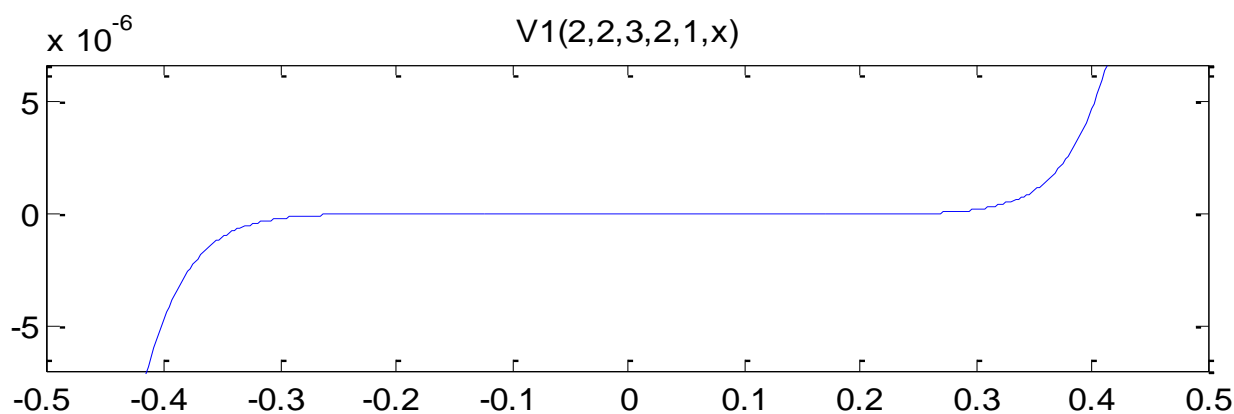

$\mathrm{V} 1(2,2,3,2,1, \mathrm{x})$

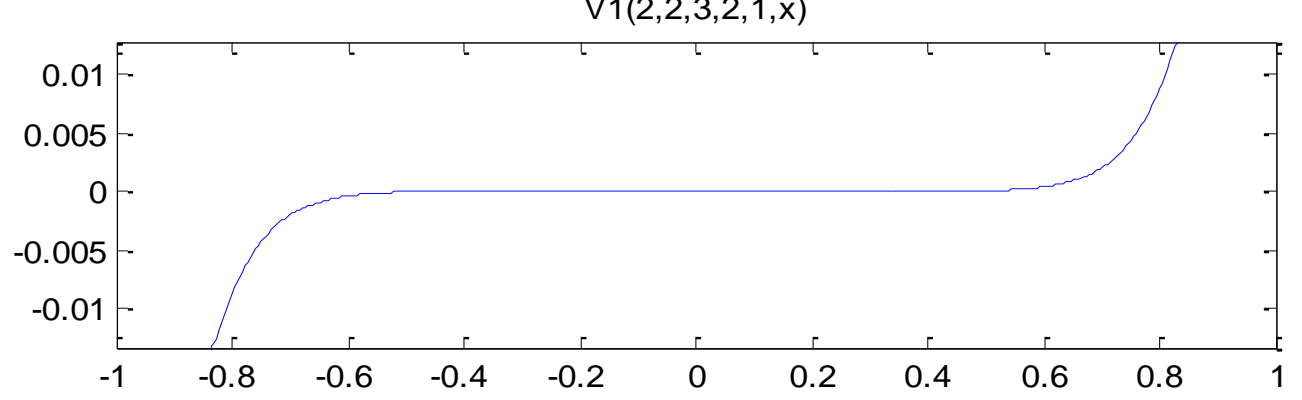

Fig. 6 Graphs of the sequence for the intervals $[-0.5,0.5]$ and $[-1,1]$ for $n=1$ 

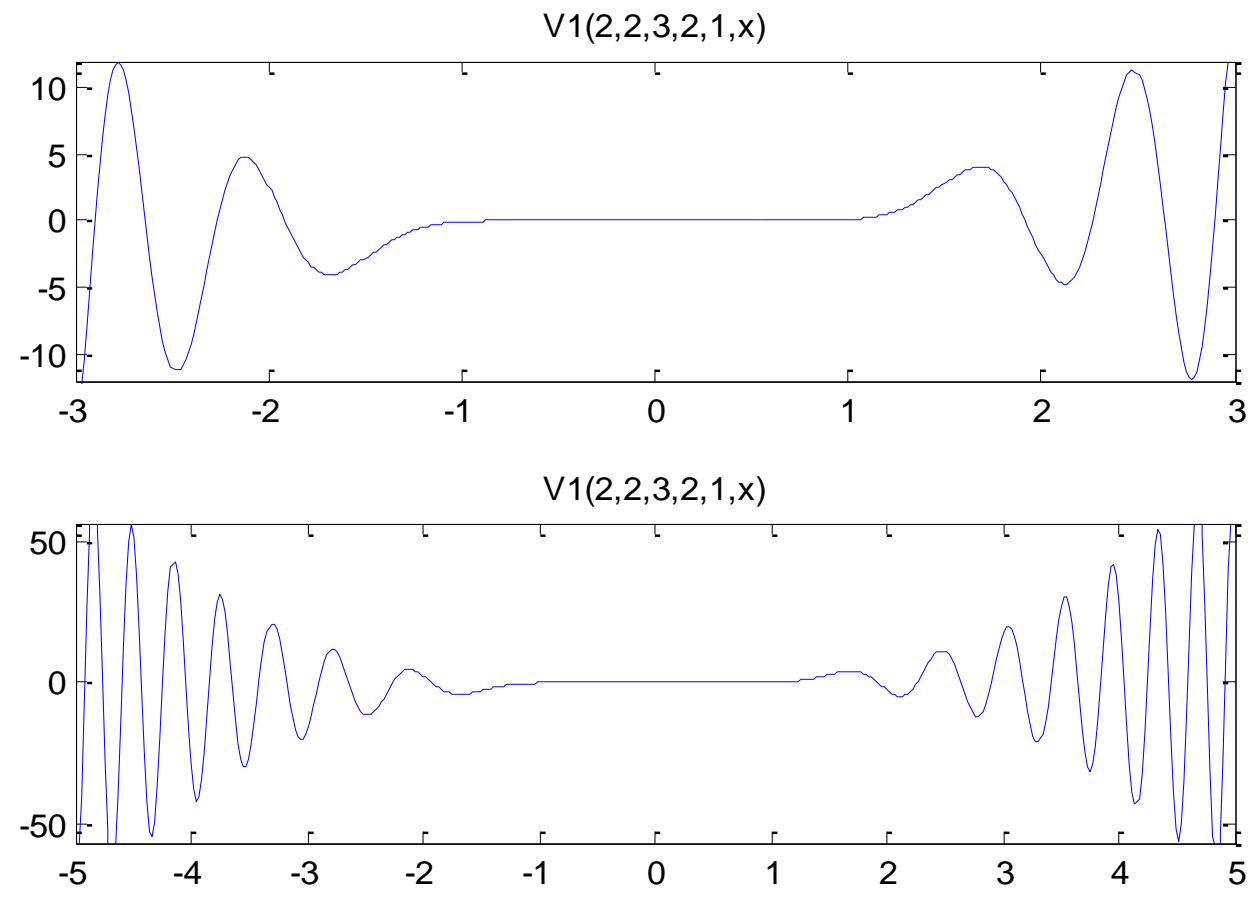

Fig. 7 Graphs of the sequence for the intervals $[-3,3]$ and $[-5,5]$ for $n=1$
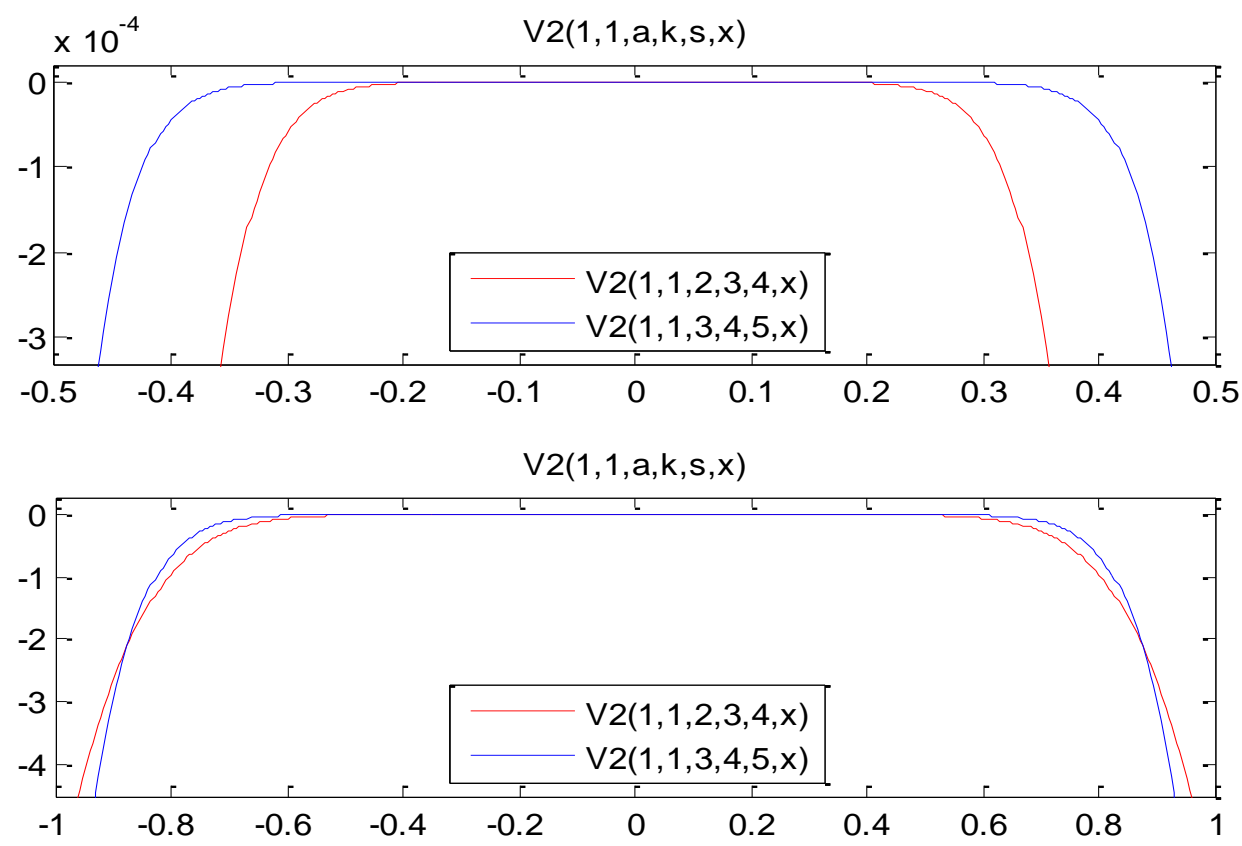

Fig. 8 Graphs of the sequence for the intervals $[-0.5,0.5]$ and $[-1,1]$ for $n=2$ 

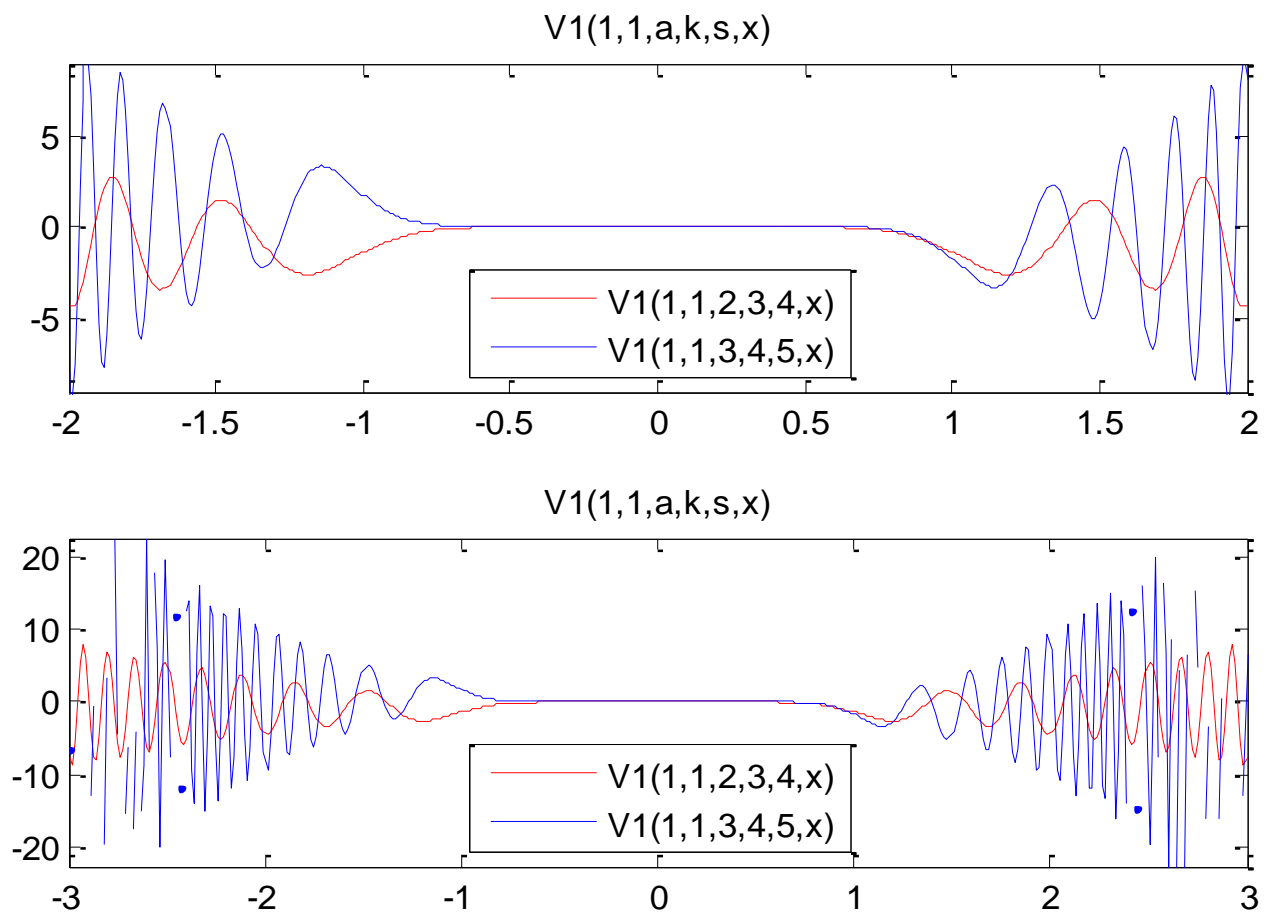

Fig. 9 Graphs of the sequence for the intervals $[-2,2]$ and $[-3,3]$ for $n=1$

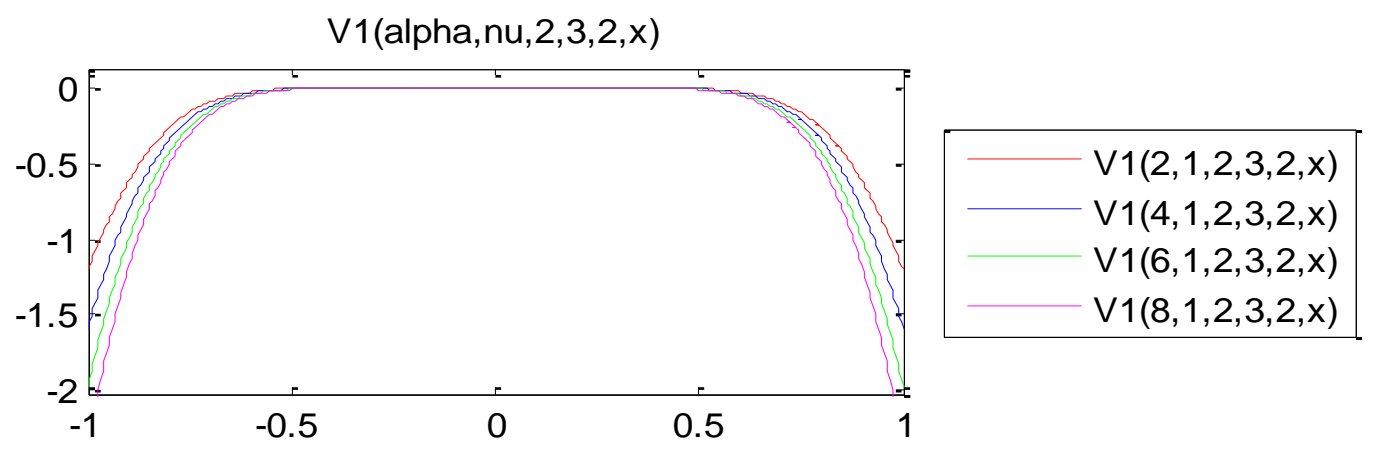

V1(alpha,nu,2,3,2,x)

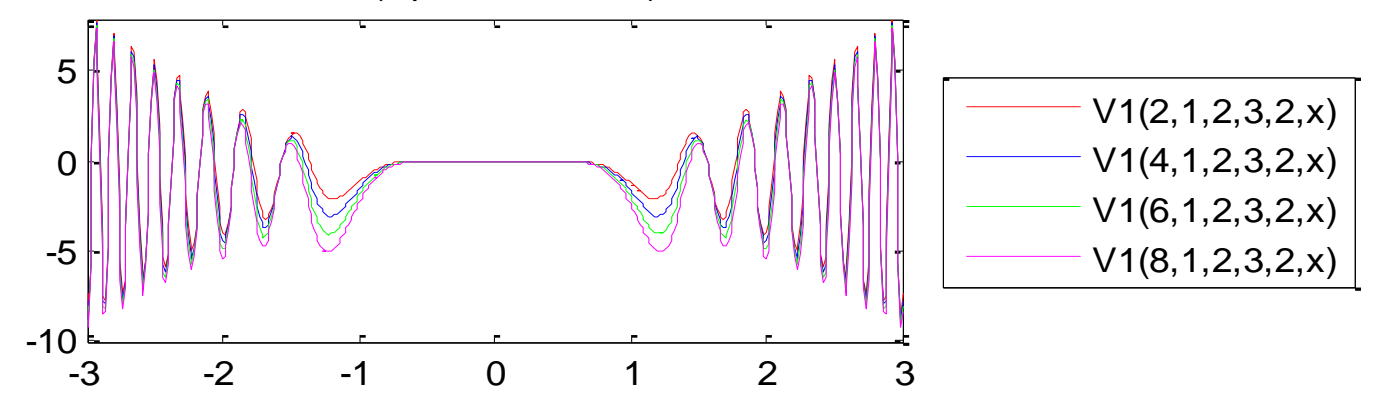

Fig. 10 Graphs of the sequence for the intervals $[-1,1]$ and $[-3,3]$ for $n=1$ 

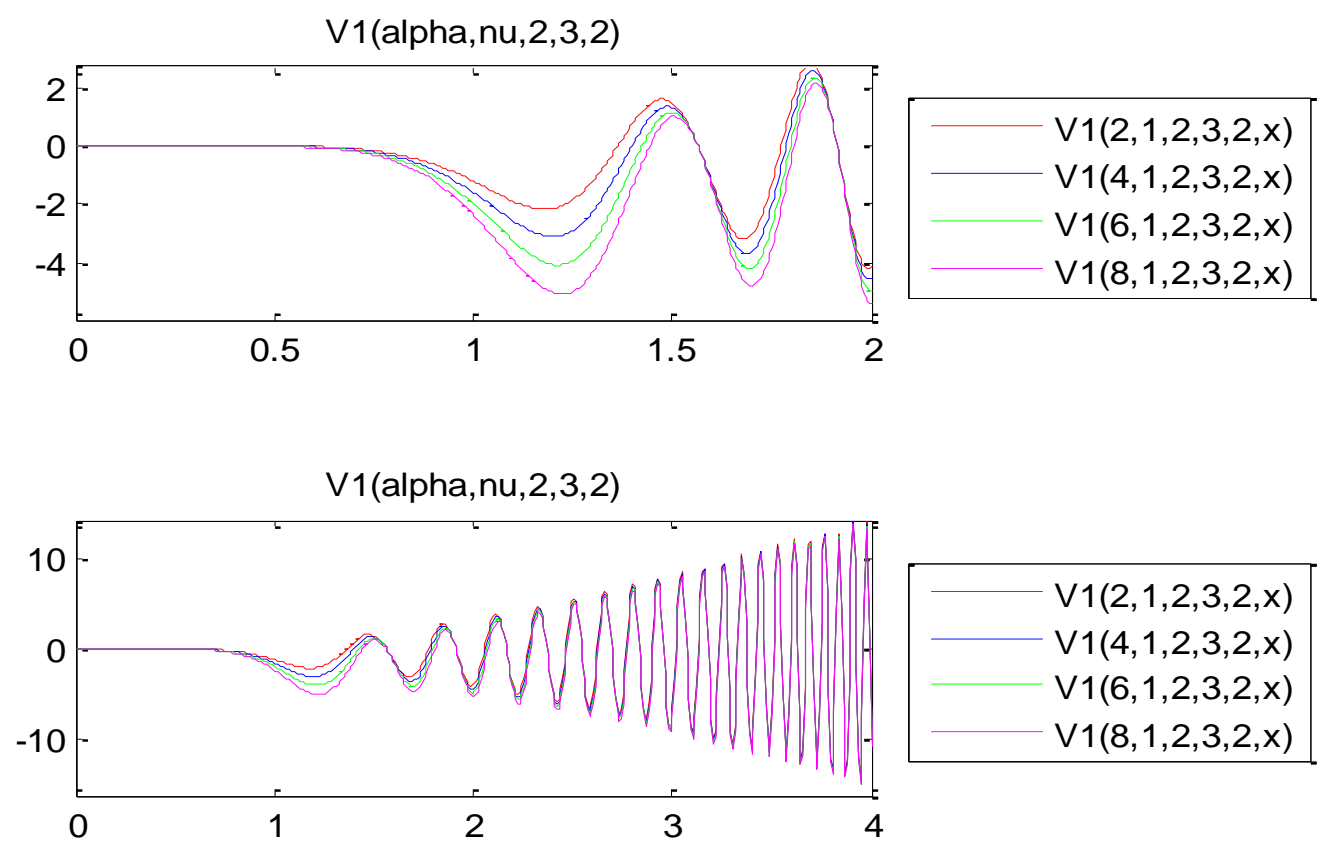

Fig. 11 Graphs of the sequence for the intervals $[0,2]$ and $[0,4]$ for $n=1$

\section{INTERPRETATION OF THE ABOVE DATABASE AND GRAPHS}

The different values of the parameters, as assigned for equation (1.15) are taken to establish different databases and their graphs. In this section interpretation of the above database and graphs are made for different parameters of the new sequence of functions.

(I) The first database and graph of the sequence of functions $\operatorname{Vn}(1,1,1,1,1, \mathrm{x})$ for $\mathrm{n}=1,2,3$ and 4 are established for the range $-2 \leq x \leq 2$. From the database (1) and Figure (1), it is easily observed that $\mathrm{V} 1(1,1,1,1,1, \mathrm{x})$ and $\mathrm{V} 3(1,1,1,1,1, \mathrm{x})$ are anti-symmetric in the interval about the line $\mathrm{x}=0$ whereas $\mathrm{V} 2(1,1,1,1,1, \mathrm{x})$ and $\mathrm{V} 4(1,1,1,1,1, \mathrm{x})$ are symmetric about the line $\mathrm{x}=0$ for the given interval.

(II) The second database and graph of the sequence of functions $\operatorname{Vn}(-1,2,1,2,1, \mathrm{x})$ for $\mathrm{n}=1,2,3$ and 4 are established for the range $-2 \leq x \leq 2$. From the database (2) and Figure (2), it is easily observed that V1($1,2,1,2,1, \mathrm{x})$ and $\mathrm{V} 3(-1,2,1,2,1, \mathrm{x})$ are anti-symmetric in the interval about the line $\mathrm{x}=0$ whereas $\mathrm{V} 2(-1,2,1,2,1, \mathrm{x})$ and $\mathrm{V} 4(-1,2,1,2,1, \mathrm{x})$ are symmetric about the line $\mathrm{x}=0$ for the given interval.

(III) The third database and graph of the sequence of functions V1(alpha,2,3,2,1,x) for alpha=2, 4, 6, 8 are established for the range $-2 \leq x \leq 2$. From the database (3) and Figure (3), it is easily observed that $\mathrm{V} 1(2,2,3,2,1, \mathrm{x}), \mathrm{V} 1(4,2,3,2,1, \mathrm{x}), \mathrm{V} 1(6,2,3,2,1, \mathrm{x})$ and
$\mathrm{V} 1(8,2,3,2,1, \mathrm{x})$ are anti-symmetric about the line $\mathrm{x}=0$ for the given interval.

(IV) The fourth database and graph of the sequence of functions V2(alpha,2,3,2,1,x) for alpha $=2,4,6,8$ are established for the range $-2 \leq x \leq 2$. From the database (4) and Figure (4), it is easily observed that $\mathrm{V} 2(2,2,3,2,1, \mathrm{x}), \mathrm{V} 2(4,2,3,2,1, \mathrm{x}), \mathrm{V} 2(6,2,3,2,1, \mathrm{x})$ and $\mathrm{V} 2(8,2,3,2,1, \mathrm{x})$ are symmetric about the line $\mathrm{x}=0$ for the given interval.

(V) The fifth database and graph of the sequence of functions $\mathrm{V} 2(1,1, \mathrm{a}, \mathrm{k}, \mathrm{s}, \mathrm{x})$ for different values of the parameters are established for the range $-0.5 \leq x \leq 0.5$. From the database (5) and Figure (5), it is easily observed that $\mathrm{V} 2(1,1,2,3,4, \mathrm{x}), \quad \mathrm{V} 2(1,1,3,4,5, \mathrm{x}), \quad \mathrm{V} 2(1,1,4,5,6, \mathrm{x})$ and $\mathrm{V} 2(1,1,5,6,7, \mathrm{x})$ are symmetric about the line $\mathrm{x}=0$ for the given interval. The graph of the sequence for all the parameters lies below the line $\mathrm{y}=0$ in the interval $-0.5 \leq x \leq 0.5$.

(VI) In the figure 6 and 7 for the sequence $\mathrm{V} 1(2,2,3,2,1, \mathrm{x})$, where $x$ lies in the intervals $[-0.5,0.5],[-1,1],[-3,3]$ and $[-5,5]$; it is observed that graphs for the said range of the sequence of function are anti-symmetric. The figures 8 and 9 of the sequences $\mathrm{V} 1(1,1,2,3,4, \mathrm{x})$ and $\mathrm{V} 1(1,1,3,4,5, \mathrm{x})$ for the intervals $[-0.5,0.5],[-1,1],[-2,2]$ and $[-3,3]$ are also anti-symmetric. Figure 10 and 11 of the sequences V1(alpha, 1,2,3,2,x) where alpha=2,4,6,8; for the interval $[-1,1],[-3,3],[0,2]$ and $[0,4]$ also can be easily interpreted. 


\section{CONCLUSION}

This paper presents several cases of MATLAB applications in mathematical analysis including generating the graph and database of sequence of function. Program made for sequence of function is easy to establish and implementation for Graphs and Data-Bases. Authors come to the conclusion on the basis of above interpretation that the sequence of functions is either symmetric or anti-symmetric for different values of the parameters for even and odd values of $n$ in particular. The present paper has enabled to find the values of sequence of functions for different values of parameters and have paved the way for comparison of trends.

\section{REFERENCES}

[1] Chak, A. M., 1956, A class of polynomials and generalization of stirling numbers, Duke J. Math., 23, 4555.

[2] Chandel, R.C.S., 1973, A new class of polynomials, Indian J. Math., 15(1), 41-49.

[3] Chandel, R.C.S., 1974, A further note on the class of polynomials $T_{n}^{\alpha, k}(x, r, p)$,Indian J. Math., 16(1), 3948.

[4] Chatterjea, S. K., 1964, On generalization of Laguerre polynomials, Rend. Mat. Univ. Padova, 34, 180-190.

[5] Craven Thomas and Csordas George, 2006, The FoxWright functions and Laguerre multiplier sequences, J. Math. Anal. and Appl. 314, 109-125.

[6] Dunn, Peter K., Understanding statistics using computer demonstrations. Journal of Computers in Mathematics and Science Teaching, 22 (3). pp. 261-281. ISSN 07319258, 2003

[7] Gould, H. W. and Hopper, A. T., 1962, Operational formulas connected with two generalizations of Hermite polynomials, Duck Math. J., 29, 51-63.

[8] J. J. O' Connor and R. E. F., Friedrich Wilhelm Bessel (School of Mathematics and Statistics University of St Andrews Scotland, 1997).

[9] Jennifer Niedziela, Bessel Functions and Their Applications, University of Tennessee-Knoxville, (Dated: October 29, 2008).
[10] Joshi, C. M. and Prajapat, M. L., 1975, The operator $T_{a, k}$ , and a generalization of certain classical polynomials, Kyungpook Math. J., 15, 191-199.

[11] Mittal, H. B., 1971, A generalization of Laguerre polynomial, Publ. Math. Debrecen, 18, 53-58.

[12] Mittal, H. B., 1971, Operational representations for the generalized Laguerre polynomial, Glasnik Mat.Ser III, 26(6), 45-53.

[13] Mittal, H. B., 1977, Bilinear and Bilateral generating relations, American J. Math., 99, 23-45.

[14] Patil, K. R. and Thakare, N. K., 1975, Operational formulas for a function defined by a generalized Rodrigues formula-II, Sci. J. Shivaji Univ. 15, 1-10.

[15] Rainville, E.D.,1971 Special functions, Chelsea Publishing Company, Bronx, New York.

[16] Shampine, L. F., Robert Ketzscher, Using AD to solve BVPs in MATLAB Journal ACM Transactions on Mathematical Software, Volume 31 Issue 1, ACM New York, NY, USA, March 2005.

[17] Singh, R. P., 1968, On generalized Truesdell polynomials, Rivista de Mathematica, 8, 345-353.

[18] Srivastava, A. N. and Singh, S. N., 1979, Some generating relations connected with a function defined by a Generalized Rodrigues formula, Indian J. Pure Appl. Math., 10(10), 1312-1317.

[19] Srivastava, H. M. and Singhal, J. P., 1971, A class of polynomials defined by generalized Rodrigues formula, Ann. Mat. Pura Appl., 90(4), 75-85.

[20] Shrivastava, P. N., 1974, Some operational formulas and generalized generating function, The Math. Education, 8, 19-22.

[21] Shukla, A. K. and Prajapati J. C., 2007, On some properties of a class of Polynomials suggested by Mittal, Proyecciones J. Math., 26(2), 145-156.

[22] Stephen, M. Watt, Making Computer Algebra More Symbolic (Invited), pp. 43-49, Proc. Transgressive Computing: A conference in honor or Jean Della Dora, (TC 2006), Granada Spain, April 24-26 2006. 